\title{
Time-Step Considerations in Particle Simulation Algorithms for Coulomb Collisions in Plasmas
}

\author{
Bruce I. Cohen, Andris M. Dimits, Alex Friedman, and Russel E. Caflisch
}

\begin{abstract}
The accuracy of first-order Euler and higher-order time-integration algorithms for grid-based Langevin equations collision models in a specific relaxation test problem is assessed. We show that statistical noise errors can overshadow time-step errors and argue that statistical noise errors can be conflated with time-step effects. Using a higher-order integration scheme may not achieve any benefit in accuracy for examples of practical interest. We also investigate the collisional relaxation of an initial electron-ion relative drift and the collisional relaxation to a resistive steady-state in which a quasi-steady current is driven by a constant applied electric field, as functions of the time step used to resolve the collision processes using binary and grid-based, testparticle Langevin equations models. We compare results from two grid-based Langevin equations collision algorithms to results from a binary collision algorithm for modeling electron-ion collisions. Some guidance is provided on how large a time step can be used compared to the inverse of the characteristic collision frequency for specific relaxation processes.
\end{abstract}

Index Terms-Algorithms, collision processes, computer applications, numerical analysis, particle collisions, plasmas.

\section{INTRODUCTION}

$\mathbf{T}$ HERE are two popular types of algorithms for Coulomb collisions in particle simulations of plasmas using finitesized particles and deposition of charge and current densities onto a grid (particle-in-cell simulation, i.e., PIC simulation). In the binary algorithm, particles in a subdomain, e.g., a cell, are grouped into discrete pairs of interacting particles such that the relative velocity is scattered through an angle whose statistical variance is dictated by the theory of Coulomb collisions in a plasma in the Fokker-Planck limit [1], [2]. The postcollision velocities of the interacting pair conserve momentum and energy relative to the pre-collision velocities. In the second type of algorithm, the collisions are modeled by defining test and field particles. The test-particle velocity is subject to drag and diffusion in three velocity dimensions using Langevin equations whose drag and diffusion coefficients depend jointly

Manuscript received November 13, 2009; revised March 29, 2010; accepted April 14, 2010. Date of publication June 1, 2010; date of current version September 10, 2010. This work was performed under the auspices of the U.S. Department of Energy by the Lawrence Livermore National Laboratory under Contract DE-AC52-07NA27344 and under Grant DE-FG02-05ER-25710 at University of California at Los Angesles under the Multiscale Initiative program supported by the DOE Office of Scientific Computing Research.

B. I. Cohen, A. M. Dimits, and A. Friedman are with the Lawrence Livermore National Laboratory, Livermore, CA 94551 USA (e-mail: cohen1@ 1lnl.gov; dimits1@1lnl.gov; afriedman@lbl.gov).

R. E. Caflisch is with the Mathematics Department, University of California at Los Angeles, Los Angeles, CA 90024 USA (e-mail: caflisch @ math.ucla.edu).

Color versions of one or more of the figures in this paper are available online at http://ieeexplore.iee.org.

Digital Object Identifier 10.1109/TPS.2010.2049589 on the velocity of the test particle and the moments of the fieldparticle velocity distribution deposited on the configurationspace mesh [3]-[7]. The grid-based Langevin equations model conserves the particle number trivially and conserves energy and momentum approximately in a statistical sense after averaging over many collisions and over the velocity distribution functions, although energy and momentum conservation can be repaired by scaling and shifting velocities after the Monte Carlo collisions occur on each time step. The drag and diffusion coefficients are derived from the classical theory of screened Coulomb collisions in the Fokker-Planck limit [4], [8]-[10]. It is of practical interest to assess the accuracy of the time integration of the collisional evolution of the plasma velocity distribution using these algorithms.

We investigate the accuracy issues for first-order Euler and higher-order time-integration algorithms for two grid-based Langevin equations collision models for a specific relaxation test problem. In an example of practical interest using numerical parameters that are typical for plasma simulations, we find that statistical noise errors can dominate systematic timestep errors. We then argue that statistical noise errors can be conflated with time-step effects. Moreover, we find that using a higher-order integration scheme may achieve no benefit in accuracy. We also find that when a higher-order Milstein correction [11], [12] to the Langevin equations model is included, there is no significant change in the results of the collisional relaxation process for the specific example considered. Results using the binary collision algorithm for the same collisional relaxation test problem have been reported by Wang et al. [13]. In the Wang et al. study, it was found that the mixing of statistical errors with time-step effects made it difficult to obtain unambiguous and clear scalings of the errors with respect to the time step used. Here, we do not undertake a detailed convergence analysis of the grid-based Langevin-equation collision algorithm with respect to the time step and the particle number. Instead, we limit ourselves to the examination of the influence of changing the time step on the results of simulations using two variants of the grid-based Langevin equations collision algorithm for numbers of particles that are typical of those used in well-resolved plasma physics studies.

We also investigate the collisional relaxation of an initial electron-ion relative drift and the relaxation to a resistive steady-state in which a quasi-steady current is driven by a constant applied electric field, as functions of the time step used to resolve the collision processes. We show that one of the two grid-based Langevin equations models investigated has an unfavorable mass-ratio scaling such that modeling electronion collisions can require a much smaller time step than that 
required using either the binary collision algorithm or a second Langevin equations algorithm that employs spherical polar velocity coordinates [6] in the example studied.

This paper is organized as follows. In Section II we provide brief overviews of the binary and test-particle, Langevinequations Coulomb collision algorithms used in particle codes. We also provide some pertinent discussions of their properties. In Section III, we present results from simulations using the test-particle Langevin equations and the grid-based Coulomb collision algorithms in which we have employed either a firstorder Euler integration, a higher-order predictor-corrector time integration, or a first-order Euler integration including the Milstein correction. In Section IV, we show simulation results using the binary collision algorithm to study the collisional relaxation of a relative drift between electron and ion species. We also present results for the collisional relaxation to a resistive steady state given a constant electric field using both binary and test-particle Langevin-equations Coulomb collision algorithms. A brief summary is presented in Section V. The findings here are intended to be of practical value to computational plasma physicists undertaking kinetic simulations in which Coulomb collisions are included. The findings here also intend to give insight into the behavior of the collision algorithms considered here.

\section{Collision Algorithms in Particle Codes}

The use of grid-based Langevin equations to model Coulomb collisions is well established [3]-[7], [10]. The algorithms are based on the classic theory describing screened Coulomb collisions in the Fokker-Planck limit [8], [9], [14], which yields the ensemble-averaged drag and diffusion coefficients $\langle\Delta \mathbf{v} / \Delta t\rangle$ and $\langle\Delta \mathbf{v} \Delta \mathbf{v} / \Delta t\rangle$. The algorithm for a velocitydependent Langevin equations collision operator in an isotropic Maxwellian background plasma can be represented in a convenient approximation [7] as follows for a test particle with velocity $\mathbf{v}$ in the local mean drift frame of the background field particles:

$$
\begin{aligned}
\Delta v_{z} & =F_{d} \Delta t+g(v) \Delta t^{1 / 2} N_{1} \\
g(v) & =\left[A_{D} G\left(\frac{v}{\sqrt{2} v_{\mathrm{th}, f}}\right) / \mathrm{v}\right]^{1 / 2} \\
F_{d} & =-\left(m_{f} / 2 T_{f}\right) A_{D}\left(1+m_{t} / m_{f}\right) G \\
A_{D} & =8 \pi n_{f} q_{t}^{2} q_{f}^{2} \ln \Lambda / m_{t}^{2} \quad G(u) / u \approx \frac{1}{2} \frac{1}{u^{3}+\frac{3 \sqrt{\pi}}{4}} \\
u & =v_{t} / \sqrt{2} v_{\mathrm{th}, f}, \quad v_{\mathrm{th}, f}=\left(T_{f} / m_{f}\right)^{1 / 2} \\
\Delta v_{\perp 1,2} & =\left[A_{D}(\Phi-G) / v\right]^{1 / 2} \Delta t^{1 / 2} N_{2,3}, \quad \Phi=\operatorname{erf}(u) \\
N_{1,2,3} & \equiv \text { Gaussian random nos. } \\
\left\langle N_{i}\right\rangle & =0, \quad\left\langle N_{i}^{2}\right\rangle=1
\end{aligned}
$$

where $\ln \Lambda$ is the Coulomb logarithm, erf is the error function, and the subscripts $t$ and $f$ are the test and field particles, respectively. The $z$-axis is aligned with the velocity of the test particle before the collision. Equation (1) describes the velocity increments $\Delta v_{z}$ and $\Delta v_{\perp 1,2}$ (in the $z$-direction and the two binormal directions) acquired by the test particles due to collisions with the field particles in the drift frame of the field particles. The $z$-axis in (1) coincides with the velocity vector of the test particle in the local mean drift frame of the field particles before the test particle is collisionally scattered. The scattered velocity vector is transformed back to the laboratory Cartesian frame with the rotation matrix given in [1] or [6], and the local mean drift of the field particles is added. Equation (1) is a discretized solution of the Fokker-Planck equation for the probability density of velocities $f(\mathbf{v})$

$$
\begin{aligned}
\frac{\partial}{\partial t} f(\mathbf{v}) & =-\frac{\partial}{\partial \mathbf{v}} \cdot\left[\mathbf{F}_{d}(\mathrm{v}) f(\mathbf{v})\right]+\frac{1}{2} \frac{\partial^{2}}{\partial \mathbf{v} \partial \mathbf{v}} \cdot[\mathbf{D}(v) f(v)] \\
\mathbf{F}_{d} & =\langle\Delta \mathbf{v} / \Delta t\rangle, \quad \mathbf{D}=\langle\Delta \mathbf{v} \Delta \mathbf{v} / \Delta t\rangle .
\end{aligned}
$$

In (1), we have corrected a minor typographical error that appeared in the approximation to $G$, which was introduced by Sherlock in (16)-(18) in his publication. [7] In the general circumstance in which the background field particle's velocity distribution function is not an isotropic Maxwellian, Rosenbluth potentials must be constructed; consequently, the collision operator acquires more structure. [4], [14] In the simulations, the field particles are composed of all of the particles of a specific species, with the density, mean drift, and temperature moments of the particle velocity distribution deposited on the configuration space grid using an interpolation scheme (linear interpolation in our simulations). The collision operator (1) conserves total momentum and energy approximately if we average over all of the particles and over an ideal distribution of random numbers for all species present. In (19) of Manheimer et al., [4] a finite $\Delta t$ correction to the drag $F_{d}$ is introduced to improve energy conservation. Energy and momentum conservation can be repaired by scaling and shifting velocities after the Monte Carlo collisions occur on each time step [4], [6].

Although no magnetic field effects are included in the formulation of the Fokker-Planck collisions presented here, the collision formulation is applicable to magnetized plasmas in the following sense. In the Fokker-Planck limit [8], [9], [14], many infinitesimal small-angle collisions are assumed to occur with individual collision events whose duration $\tau$ is arbitrarily short so that the product of the acceleration due to electromagnetic Newton-Lorentz forces and the time duration $\tau$ is arbitrarily small compared to particle velocity. In this limit, the collision is unaffected by any electromagnetic fields present, although the overall particle trajectory is influenced by the electromagnetic fields. From a computational perspective, this invites the use of operator splitting to accommodate both the collisions and the Newton-Lorentz forces due to electromagnetic fields. The result of the collision should be insensitive to when during the time step the collision event occurs.

A variant of the grid-based Langevin-equations Coulomb collision operator has been introduced by Lemons et al. [6]. The methodology in [6] scatters the velocity vector of the test particle using spherical polar coordinates $(v, \theta, \phi)$. The magnitude of the velocity is subject to both drag and diffusion, and the polar angle is subject to diffusion. There is an advantage in using spherical coordinates for scattering processes that are 
dominantly pitch-angle scattering at nearly constant energy of the test particle, e.g., scattering of a light particle by a very heavy particle. We will illustrate this in the following discussion and example calculations. The Langevin equations in [6] are repeated here for convenience

$$
\begin{aligned}
\Delta \mathrm{v}= & F_{v} \Delta t+g(\mathrm{v}) \Delta t^{1 / 2} N_{1}, \\
g(\mathrm{v})= & {\left[A_{D} G\left(\frac{\mathrm{v}}{\sqrt{2} \mathrm{v}_{t h, f}}\right) / \mathrm{v}\right]^{1 / 2} } \\
F_{v}= & -\left(A_{D} / 2 \mathrm{v}^{2}\right) \\
& \times\left[\left\{\left(1+m_{t} / m_{f}\right) \mathrm{v}^{2} / \mathrm{v}_{t h, f}^{2}+1\right\} G-\Phi\right], \\
A_{D}= & 8 \pi n_{f} q_{t}^{2} q_{f}^{2} \ln \Lambda / m_{t}^{2} \\
G(u) / u \approx & \frac{1}{2} \frac{1}{u^{3}+\frac{3 \sqrt{\pi}}{4}}, \quad \Phi=\operatorname{erf}(u), \\
u= & \mathrm{v}_{t} / \sqrt{2} \mathrm{v}_{t h, f}, \quad \mathrm{v}_{t h, f}=\left(T_{f} / m_{f}\right)^{1 / 2} \\
\Delta \theta= & {\left[A_{D}(\Phi-G) / \mathrm{v}^{3}\right]^{1 / 2} \Delta t^{1 / 2} N_{2} } \\
\Delta \phi= & 2 \pi U[0,1] \\
N_{1,2} \equiv & \text { Gaussian random nos., } \\
<N_{i}>= & 0, \quad<N_{i}^{2}>=1, \\
U \equiv & \equiv \text { uniform random no., } \quad 0 \leq U \leq 1 .
\end{aligned}
$$

At low test-particle energies, $F_{v}$ and $\Delta \theta$ diverge in (3). To resolve the divergence, Lemons et al. [6] retain the dominant terms in the expression for the change in the speed; thus, they derive $v(t+\Delta t)=\sqrt{v(t)^{2}+(4 / 3 \sqrt{2 \pi}) A_{D} \Delta t / v_{\mathrm{th}, f}}$ for $v^{2} \leq(4 / 3 \sqrt{2 \pi}) A_{D} \Delta t / v_{\text {th }, f}$. However, $\Delta \theta^{2} \propto 1 / v^{2}, \Delta v_{\perp}^{2} \propto$ $v^{2} \Delta \theta^{2}$ is not divergent.

An algorithm for binary Coulomb collisions has been introduced in the classic work of Takiziuka and Abe [1]. In the binary algorithm, equally weighted particles in a cell are paired and then the relative velocity vector of the two particles is scattered through a random scattering angle with variance dictated by the theory of screened Coulomb collisions in a plasma [8], [9]. After the relative velocity vector is scattered, the two scattered particle velocities are reconstructed such that particle momentum and energy are conserved algebraically. Particle number is conserved identically. Nanbu [2] extended the algorithm of Takizuka and Abe [1] to allow for a larger time step by aggregating multiple collisions. In Takizuka and Abe [1], the relative velocity of a pair is scattered through an angle $\Theta$ with variance related to

$$
\begin{aligned}
\left\langle\delta^{2}\right\rangle & =2 \pi q_{1}^{2} q_{2}^{2} n_{(1,2)<} \ln \Lambda \Delta t / m_{12}^{2} u^{3} \\
u & =\left|\mathbf{v}_{1}-\mathbf{v}_{2}\right|, \quad m_{12}=m_{1} m_{2} /\left(m_{1}+m_{2}\right), \quad \delta \equiv \tan \frac{\Theta}{2}
\end{aligned}
$$

and through a random angle $\phi$ about the axis of the relative velocity before the scattering event. The post-collision velocity vectors of the scattered pair are constructed from the scattered relative velocity vector. There is no separation of test and field particles in the binary scheme, and there is no assumption that the velocity distribution is isotropic and Maxwellian. However, there is an implicit assumption that the value of $\ln \Lambda$ derived for an abitrary velocity distribution (computed locally in a spatial cell) deviates insignificantly from the value of $\ln \Lambda$ derived for a Maxwellian. This method conserves particle number, energy, and momentum.

Both the grid-based Langevin equations and binary collision algorithms generalize to relativistic collisions. Both algorithms are formally accurate through $O\left(\Delta t^{1 / 2}\right)$ and both produce drag $\langle\Delta \mathbf{v} / \Delta t\rangle$ and diffusion coefficients $\langle\Delta \mathbf{v} \Delta \mathbf{v} / \Delta t\rangle$ that agree with the Spitzer-Trubnikov theory through $O(\Delta t)$ assuming perfect statistics.

The accuracy of both algorithms requires that the velocity change and the angle scattered by a test particle in one time step must be small. In the binary collision algorithm, we can easily deduce the scaling of the variance with respect to the charge state and mass for electon-electron, electron-ion, and ion-ion scattering. We note the reduced mass in (4), $m_{e e} \sim m_{e i} / 2=$ $m_{i e} / 2 \ll m_{i i}$, so that for low charge-state ions and $T_{i} \sim T_{e}$, the time step for accurately resolving the electron-electron binary collisions is comparable to that required for resolving electron-ion collisions. Further, ion-ion collisions can be resolved with a substantially larger time step. However, the gridbased Langevin equations algorithms have different numerical characteristics. From (1), one can show that the magnitudes of the drag and diffusion coefficients are monotonically increasing as the test-particle speed $\mathrm{v}$ goes to zero. In the low-velocity limit for test partcles, one can show that the perpendicular and parallel velocity diffusion coefficients for scattering a test particle on a field particle in (1) have the following scalings:

$$
\begin{aligned}
& D_{\perp, \|, e i} / D_{\perp, \|, e e}^{*} \sim Z_{i}\left(Z_{i} n_{i} / n_{e}\right) \\
& \quad \times\left(T_{e} / T_{i}\right)^{1 / 2}\left(m_{i} / m_{e}\right)^{1 / 2} \propto\left(m_{i} / m_{e}\right)^{1 / 2} \\
& D_{\perp, \|, i i} / D_{\perp, \|, e e}^{*} \sim Z_{i}^{3}\left(Z_{i} n_{i} / n_{e}\right) \\
& \quad \times\left(T_{e} / T_{i}\right)^{1 / 2}\left(m_{e} / m_{i}\right)^{3 / 2} \propto\left(m_{e} / m_{i}\right)^{3 / 2} \\
& D_{\perp, \|, i e} / D_{\perp, \|, e e}^{*} \sim Z_{i}\left(Z_{i} n_{i} / n_{e}\right) \\
& \quad \times\left(m_{e} / m_{i}\right)^{2} \propto\left(m_{e} / m_{i}\right)^{2}
\end{aligned}
$$

where the ion charge is $Z_{i} e, D_{\perp k l}=\left\langle\Delta v_{\perp} \Delta v_{\perp} / \Delta t\right\rangle$ and $D_{\| k l}=\left\langle\Delta v_{\|} \Delta v_{\|} / \Delta t\right\rangle$ for species $k$ scattering on species $l$. For $T_{i} \sim T_{e}$, however, a thermal electron has a much larger velocity than a thermal ion; thus, the drag and diffusion coefficients for electron-ion scattering should be evaluated for $v_{t} \gg v_{\mathrm{th}, f}$. In the large argument limit of (1)

$$
\begin{aligned}
D_{\|, t f} & \propto\left(n_{f} q_{t}^{2} q_{f}^{2} / m_{t}^{2} v^{3}\right) v_{\mathrm{th}, f}^{2} \\
D_{\perp, t f} & \propto\left(n_{f} q_{t}^{2} q_{f}^{2} / m_{t}^{2} v v_{\mathrm{th}, f}^{2}\right) v_{\mathrm{th}, f}^{2} \\
F_{d} & \propto-\left(n_{f} q_{t}^{2} q_{f}^{2} / m_{t}^{2} v_{\mathrm{th}, f} v^{2}\right) v_{\mathrm{th}, f} .
\end{aligned}
$$

The drag and diffusion coefficients go to zero at different rates for large test-particle velocities, and $D_{\perp, t f}$ and the $\operatorname{drag} F_{d}$ have no dependence on the mass of the field particles. However, $D_{\|, t f} \propto T_{f} / m_{f}$, and thus the parallel velocity diffusion 
coefficient for electron-ion collisions is smaller than that for electron-electron collisions by $Z_{i} m_{e} / m_{i}$ for $T_{e} \sim T_{i}$ and decreases as $1 / v^{3}$. We believe that the unfavorable mass-ratio scaling for the ratio of the electron-ion collisional diffusion to the electron-electron collisional diffusion in (5), $Z_{i}\left(m_{i} / m_{e}\right)^{1 / 2}$ at low velocities leads to the required use of a significantly smaller time step to accurately resolve the electron-ion collisions for the grid-based Langevin equations collision algorithm in (1), which is borne out in our simulation experience (illustrated in Section IV).

In the Lemons et al. algorithm for large test-particle velocities,

$$
\begin{aligned}
D_{v, t f} & \propto\left(n_{f} q_{t}^{2} q_{f}^{2} / m_{t}^{2} v^{3}\right) v_{\mathrm{th}, f}^{2} \\
D_{\perp, t f} & \propto\left(n_{f} q_{t}^{2} q_{f}^{2} / m_{t}^{2} v v_{\mathrm{th}, f}^{2}\right) v_{\mathrm{th}, f}^{2} \\
F_{v} & \propto-\left(m_{t} / m_{f}\right)\left(n_{f} q_{t}^{2} q_{f}^{2} / m_{t}^{2} v_{\mathrm{th}, f} v^{2}\right) v_{\mathrm{th}, f} .
\end{aligned}
$$

Compared to the algorithm based on (1) in the large velocity limit, the major difference is that the drag coefficient in the Lemons et al., algorithm has $F_{v} / F_{d} \propto\left(m_{t} / m_{f}\right)$; thus, the drag for electron-ion collisions is much smaller compared to the electron-electron collisions in the Lemons et al. algorithm. Furthermore, in the Lemons et al. algorithm, the scattering of the low-velocity test particles, where the drag dominates the stochastic terms, is performed to remove divergences. [8] In the limit that $m_{e} / m_{i} \rightarrow 0$, the scattering of electrons on ions should only produce angle scattering of the electron without changing the electron test-particle energy. The Lemons et al. [6] Langevin equations algorithm in (3) for electron collisions on ions is dominated by angle scattering. Moreover, the diffusion $D_{v, e i}$ in the magnitude of the electron test-particle velocity and the drag $F_{v, e i}$ are relatively weak. The slowing of the electrons on the ions comes from the angle scattering. Thus, we expect that (3) should be better able to accommodate electronion scattering; and it should allow larger time steps than the algorithm based on (1). An example of simulating electron-ion collisions is presented in Section IV.

When the collisional scattering of the velocity vector in one time step is too large using the grid-based Langevin equations algorithm based on (1), relaxation rates and energy and momentum conservation become inaccurate. If only the scattering angle $\Delta \theta$ becomes too large in (3) when the time step is too large, the angular diffusion rate becomes inaccurate, but the change in the test-particle energy may still be small. In contrast, when the time step becomes too large in the binary collision algorithm, conservation of energy and momentum is still preserved, and although relaxation rates may not be reproduced accurately, the binary collision algorithm fails gracefully.

In the work of Wang et al., [13] the convergence properties of the Takizuka and Abe and the Nanbu binary collision operators with respect to particle number and time step were studied. It was found that the Nanbu collision algorithm achieved a factorof-two improvement in relative accuracy over the Takizuka and Abe basic algorithm for the same time step. The underlying properties of the Nanbu algorithm were studied analytically in the work of Dimits et al. [15].

\section{Corrections to First-Order Euler Integration OF THE GRID-BASED LANGEVIN EQUATIONS COLLISION OPERATOR}

In this section, we report the results of the studies of the gridbased Langevin equations collision operator (1), attempting to extend it to higher-order accuracy than first-order Euler. We explore several time-discretization schemes for the grid-based Langevin equations collision operator, which are represented schematically in the following finite-difference Langevin equations fashioned after (1). In the cases considered in this section, only ion-ion collisions are considered.

First-order Euler

$$
y_{n+1}=y_{n}+\Delta t \frac{d y}{d t}\left(y_{n}\right)+N_{1} \Delta t^{1 / 2} \sqrt{D\left(y_{n}\right)} .
$$

Predictor-corrector (modified Euler)

$$
\begin{aligned}
\text { predictor : } y_{n+1}^{\prime}= & y_{n}+\Delta t \frac{d y}{d t}\left(y_{n}\right)+N_{1} \Delta t^{1 / 2} \sqrt{D\left(y_{n}\right)} \\
\text { corrector : } y_{n+1}= & y_{n}+\Delta t \frac{d y}{d t}\left(\left(y_{n+1}^{\prime}+y_{n}\right) / 2\right) \\
& +N_{2} \Delta t^{1 / 2} \sqrt{D\left(\left(y_{n+1}^{\prime}+y_{n}\right) / 2\right)} .
\end{aligned}
$$

Predictor-corrector (two-step scheme)

$$
\begin{aligned}
\text { predictor : } y_{n+1 / 2}=y_{n} & +\frac{1}{2} \Delta t \frac{d y}{d t}\left(y_{n}\right) \\
& +N_{1}\left(\frac{1}{2} \Delta t\right)^{1 / 2} \sqrt{D\left(y_{n}\right)} \\
\text { corrector : } y_{n+1}=y_{n} & +\Delta t \frac{d y}{d t}\left(y_{n+1 / 2}\right) \\
& +N_{2} \Delta t^{1 / 2} \sqrt{D\left(y_{n+1 / 2}\right)} .
\end{aligned}
$$

Partial corrector

$$
\begin{aligned}
& \text { predictor : } y_{n+1}^{\prime}=y_{n}+\Delta t \frac{d y}{d t}\left(\left(y_{n}^{\prime}+y_{n-1}^{\prime}\right) / 2\right) \\
& +N_{1} \Delta t^{1 / 2} \sqrt{D\left(\left(y_{n}^{\prime}+y_{n-1}^{\prime}\right) / 2\right)} \\
& \text { partial corrector : } y_{n+1}=y_{n}+\Delta t \frac{d y}{d t}\left(\left(y_{n+1}^{\prime}+y_{n}^{\prime}\right) / 2\right) \\
& +N_{2} \Delta t^{1 / 2} \sqrt{D\left(\left(y_{n+1}^{\prime}+y_{n}^{\prime}\right) / 2\right)}
\end{aligned}
$$

subject to the constraint relations that the ensemble averages $\left\langle\left(y_{n+1}-y_{n}\right) / \Delta t\right\rangle=d y /\left.d t\right|_{y_{n}}$ and $\left\langle\left(y_{n+1}-y_{n}\right)^{2} / \Delta t\right\rangle=$ $D\left(y_{n}\right)$ (to lowest order in powers of $\Delta t$ ) are the drag and diffusion coefficients, respectively, for the vector components of the test-particle velocities given in (1). The partially corrected Euler algorithm [16] is a special case in the family of multistep Runge-Kutta methods and is closely related to the two-stage explicit Adams method. It has advantages in that it is easy to initialize and it can be represented with a reduced 

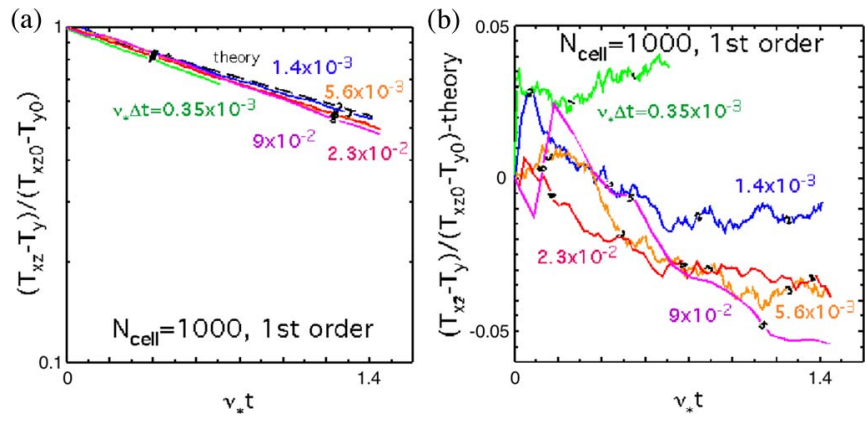

Fig. 1. BZOHAR simulations of like-species Coulomb collisions using the grid-based Langevin equations algorithm (1) with first-order Euler time integration to study the relaxation of a weak temperature anisotropy: (a) simulated temperature anisotropy versus time and (b) the difference of the simulated temperature anisotropy from theory versus time for various values of the time step (color online). (a)

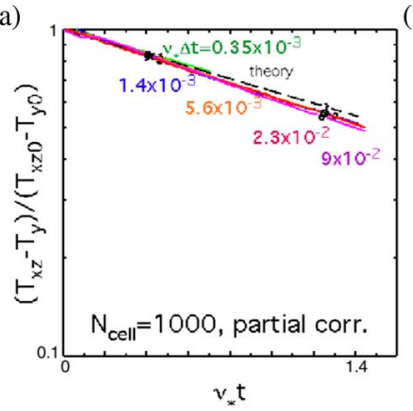

(b)

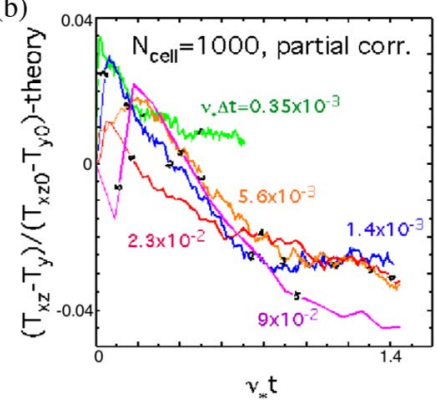

Fig. 2. BZOHAR simulations of like-species Coulomb collisions using the grid-based Langevin equations algorithm (1) with partial corrector time integration to study the relaxation of a weak temperature anisotropy: (a) simulated temperature anisotropy versus time and (b) the difference of the simulated temperature anisotropy from theory versus time for various values of the time step (color online). (a)

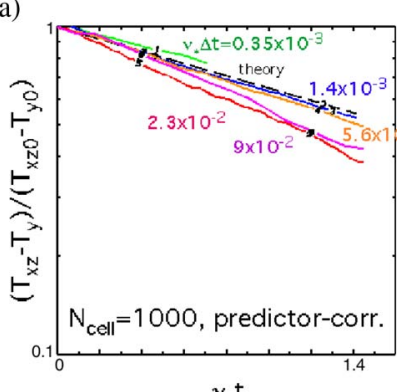

$v_{*} \mathrm{t}$ (b)

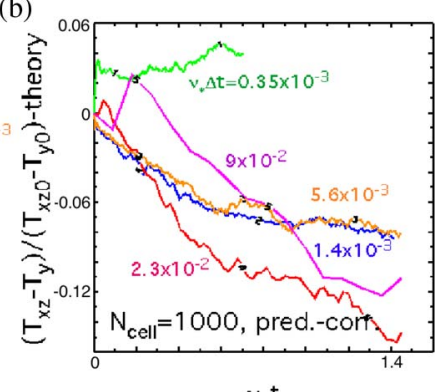

$v+t$
Fig. 3. BZOHAR simulations of like-species Coulomb collisions using the grid-based Langevin equations algorithm (1) with modified Euler predictorcorrector time integration to study the relaxation of a weak temperature anisotropy: (a) simulated temperature anisotropy versus time and (b) the difference of the simulated temperature anisotropy from theory versus time for various values of the time step (color online).

number of function evaluations: the corrector-step function evaluations can be saved for reuse in the function evaluations in the next predictor step. The method may be a natural "upgrade" for some codes using predictor-corrector methods. It is an upgrade in that it may be possible to nearly double the efficiency of the calculation even with little rearrangement of the code.

Figs. 1-3 show the results of a series of BZOHAR simulations [5] using the grid-based Langevin equations ion-ion collision operator to study the collisional relaxation of a weak ion temperature anisotropy $\left(T_{y}=0.95 T_{x}, T_{x}=T_{z}\right)$ in which we vary the product of the characteristic ion-ion collision frequency $\nu_{*}$ and the time step $\Delta t$ for 1000 particles per cell and $N_{p}=2 \times 10^{6}$ particles in one spatial dimension, where $\nu_{*}=(8 / 3 \sqrt{\pi}) \nu_{0}, \nu_{0}=\sqrt{2} \pi q^{4} n \ln \Lambda / \sqrt{m} T^{3 / 2}$ and $\nu_{0}$ is the Braginskii [20] characteristic collision frequency for likespecies collisions. In all of the BZOHAR simulations reported here, the electrons are modeled as a fluid with a Boltzmann response: the ions are particles; there are self-consistent electric fields at thermal levels and particle advection; and the grid cell size is chosen equal to the electron Debye length. The value of $\ln \Lambda$ was scaled so that the value of $\nu_{*} \Delta t$ can be set artificially. The exponential relaxation rate for a weak temperature anisotropy is given by Trubnikov [9], $\nu_{\text {relax }}=$ $(8 / 5 \sqrt{2 \pi}) \nu_{0}$. Fig. 1 shows results for the relaxation of a temperature anisotropy using the first-order Euler integration scheme, (4). Figs. 2 and 3 show the corresponding results for the first predictor-corrector algorithm (modified Euler) and the partial corrector and (9) and (11), respectively. In each of these figures, we plot the relaxation of the temperature anisotropy normalized to the initial anisotropy as a function of time and, separately, the difference of the normalized temperature anisotropy with respect to the asymptotic theory $\exp \left(-\nu_{\text {relax }} t\right)$ for several different values of $\nu_{*} \Delta t$. The number of particles per cell and the total number of particles used here are typical of those commonly used in well-resolved particle simulations of many plasma phenomena. In these simulations, we observe the weakly anisotropic Maxwellian ion velocity distributions relax by transferring energy to the colder velocity dimension from the hotter dimensions. The lower energy ions being more collisional tend to relax their temperature anisotropy faster than the more energetic ions.

The object of the scan with respect to $\nu_{*} \Delta t$ is to assess any trend in the convergence of the simulation results to the asymptotic theory. For completeness, we list several of the sources that might contribute to the deviations of the simulation results from the theory: i) the theory is asymptotic in the small parameter $\left(T_{y}-T_{x}\right) / T_{x}$, but a finite value of the initial anisotropy is used; ii) the drag and diffusion coefficients used here are calculated from an assumed isotropic Maxwellian velocity distribution, which is only an approximation to the actual weakly anisotropic distribution of test-particle velocities; iii) errors are associated with the finite value of $\nu_{*} \Delta t$ used in the discrete time integration; iv) a finite number of testparticle velocities is used to resolve the velocity distribution (and the associated random numbers used in initializing the velocity distribution of the particles); and v) there is a deviation from an ideal distribution of the finite set of random numbers associated with the finite number of collisions during any time interval. There are systematic errors associated with i), ii), and iii), and random errors associated with iv) and v). Moreover, the random errors in iv) and v) are independent of one another. However, the error analysis has an additional complication. We note that in a fixed physical time interval $\tau$, the number of collision events is determined by $\tau / \Delta t$. Hence, as we increase $\nu_{*} \Delta t$, the number of collision events and the number of random numbers $N_{r}$ associated with the collisions 
in a fixed physical time interval both decrease proportional to $\tau / \Delta t$. For finite $N_{r}$, the variance of the distribution of random numbers deviates from ideality with a standard error that scales with $1 /\left(N_{r}\right)^{1 / 2}$. Thus, there is a random error in the time-averaged diffusion rate that is expected to scale with $\Delta t^{1 / 2}$. Hence, these statistical errors and the effects of the discrete time integration are conflated. The random errors in the time-averaged diffusion rate contribute to the time histories of collisional relaxation events. The purely systematic error in the discrete time integration would be expected to scale as a power of $\Delta t$ that is higher than first order in $\Delta t$ in keeping with the behavior of a first-order Euler (or higher-order) finite-difference integration of a nonstochastic differential equation. With these scalings, the statistical noise due to iv) and v) may dominate the systematic error in the time integration iii) for any value of $\nu_{*} \Delta t$ in a particular simulation diagnostic.

Self-consistent electric fields also contribute to the total effective collisionality in these simulations due to the thermal noise and discrete particle effects [17]-[19]. An estimate for the effective collisionality due to noise fields has been given by $\mathrm{Vu}$, DuBois, and Bezzerides [18], and this estimate has been applied in the work of Cohen, $\mathrm{Vu}$, and Williams [19]. In the BZOHAR simulations for ion-ion collisions in which electric fields due to thermal noise are present, the arguments in [18] and [19] lead to an estimate for the effective ion-ion collision frequency $\nu_{i i}^{\text {eff }}$ due to electric field noise $\nu_{i i}^{\text {eff }} / \omega_{\text {pi }} \sim\left(1 / 2 n_{i} \lambda_{i}\right)\left(v / v_{i}\right)$, where $\omega_{\text {pi }}$ is the ion plasma frequency, $v_{i}$ is the ion thermal velocity, $v$ is an ion velocity, $n_{i}$ is the number of particle ions divided by the length of the system, and $\lambda_{i}$ is the ion Debye length. We will evaluate the effective ion collision frequency due to noise for a thermal ion $v=v_{i}$.

For the parameters in the simulations shown in Figs. 1-3, the effective collision frequency due to thermal noise in the electric fields is estimated to be $\nu_{i i}^{\text {eff }} \Delta t \sim 2 \times 10^{-4}$, as compared to the range of collision frequencies $3.5 \times 10^{-4} \leq \nu_{*} \Delta t \leq 9 \times 10^{-2}$ used in the simulations. Thus, only the simulations for the lowest values of $\nu_{*} \Delta t$ are affected by the additive contributions of the effective collisions due to thermal electric field fluctuations.

The simulation results in Figs. 1-3 do not show any reduction in error by using the higher-order-accurate partial corrector or predictor-corrector (modified Euler) algorithms. If there are only systematic discrete-time-integration errors associated with $\nu_{*} \Delta t$, then one might expect a scaling of the systematic time-step errors proportional to $\left(\nu_{*} \Delta t\right)^{r}$, where $r>1$ for the first-order Euler algorithm and proportional to a higher power of $\nu_{*} \Delta t$ for the higher order partial-corrector and predictorcorrector algorithms based on the behavior of integrating nonstochastic differential equations. However, we observe no linear or higher order scaling of the errors with respect to $\nu_{*} \Delta t$. The superposition of random and systematic discrete-timeintegration errors in the stochastic diffusion terms obscures deducing any clean error scaling with respect to the time step. The comparisons of the simulation results with theory do show a degradation of the agreement when the value of $\nu_{*} \Delta t$ becomes sufficiently large.

A lack of a clear scaling with time step is also observed for the binary collision algorithm reported in the work of Wang et al. [13]. In [13], the binary collison models of [1] and [2]
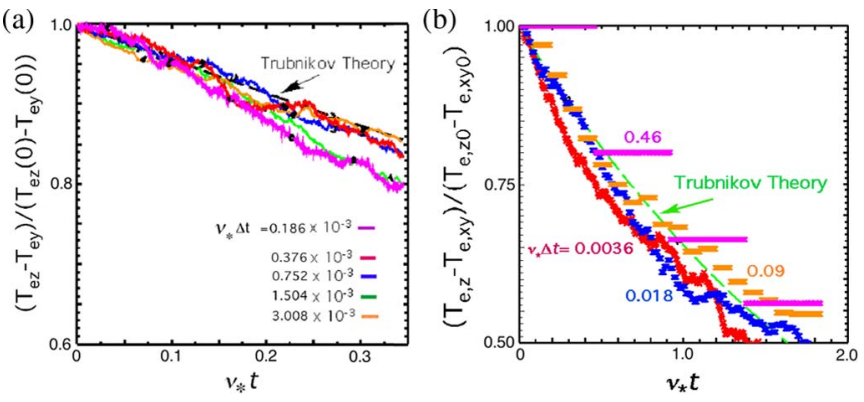

Fig. 4. Particle simulations suppressing the electric field and particle advection of like-species Coulomb collisions using the grid-based Langevin equations algorithms with first-order Euler time integration to study the relaxation of a weak temperature anisotropy: simulated temperature anisotropy versus time for various values of the time step and algorithms based on (a) (1) and (b) (3) (color online).

are employed, and convergence with respect to particle number and time step are investigated with the additional feature that ensemble averages are computed over many realizations of the simulations to reduce the statistical variance. For fixed time step, the simulations in [13] show a convergence with respect to increasing the number of particles or the number of realizations, i.e., the variance decreases as $1 / N$, where $N$ is the product of the number of particles and realizations. The scaling of the errors with respect to $\Delta t$ is less clear, but some cases show a rough error scaling approximately as $\Delta t^{1 / 2}$ in Fig. 5 of [13].

In Fig. 4, we show results for a series of simulations using the first-order Euler grid-based Langevin equations collision operators, combining (1) and (8) in Fig. 4(a) and using the Lemons et al. algorithm (3) and (8) in Fig. 4(b), with electric fields and particle advection suppressed. The initial condition here corresponds to a weak temperature anisotropy $\left(T_{y}=\right.$ $\left.0.95 T, T_{x}=T_{z}=1.05 T\right)$. In the series of simulations, we vary the product of the characteristic collision frequency $\nu_{*}$ and the time step $\Delta t$ for 600 particles per cell and $N_{p}=6 \times 10^{4}$ in one spatial dimension. A single species scatters on itself to relax its temperature anisotropy here. We varied $\nu_{*} \Delta t$ over a range of values $\nu_{*} \Delta t \leq 3 \times 10^{-3}$ in Fig. 4(a) and $\nu_{*} \Delta t \leq 0.46$ in Fig. 4(b). We observe some random scatter in the relaxation of the temperature anisotropy and no systematic dependence on $\nu_{*} \Delta t$ over the range of $\nu_{*} \Delta t$ values used in both Fig. 4(a) and (b). The relaxation of the temperature anisotropy depends primarily on the angle scattering of the test-particle velocity vector, for which both of the collision algorithms in (1) and (3) for single species collisions perform relatively well at significant values of the $\nu_{*} \Delta t$, i.e., $\nu_{*} \Delta t<0.1$ in the examples shown here (Figs. 1-6).

In Fig. 5, we compare the results of simulation scans using the grid-based Langevin equations collision algorithm (1) and the first-order Euler and the second predictor-corrector (two step) integration schemes, (8) and (10), respectively, for likespecies collisional relaxation of a weak temperature anisotropy $\left(T_{y}=0.95 T_{x}, T_{x}=T_{z}\right)$ and extending to larger values of $\nu_{*} \Delta t$ than those in Figs. 1-3. In these simulations, there are 333 particles per cell and $N_{p}=666000$ in one spatial dimension, and $\nu_{*} \Delta t$ is varied in the series. The influence of thermal electric field fluctuations on the collisionality here is small at $\nu_{i i}^{\text {eff }} \Delta t \sim 3.5 \times 10^{-4}$ because this is small compared 

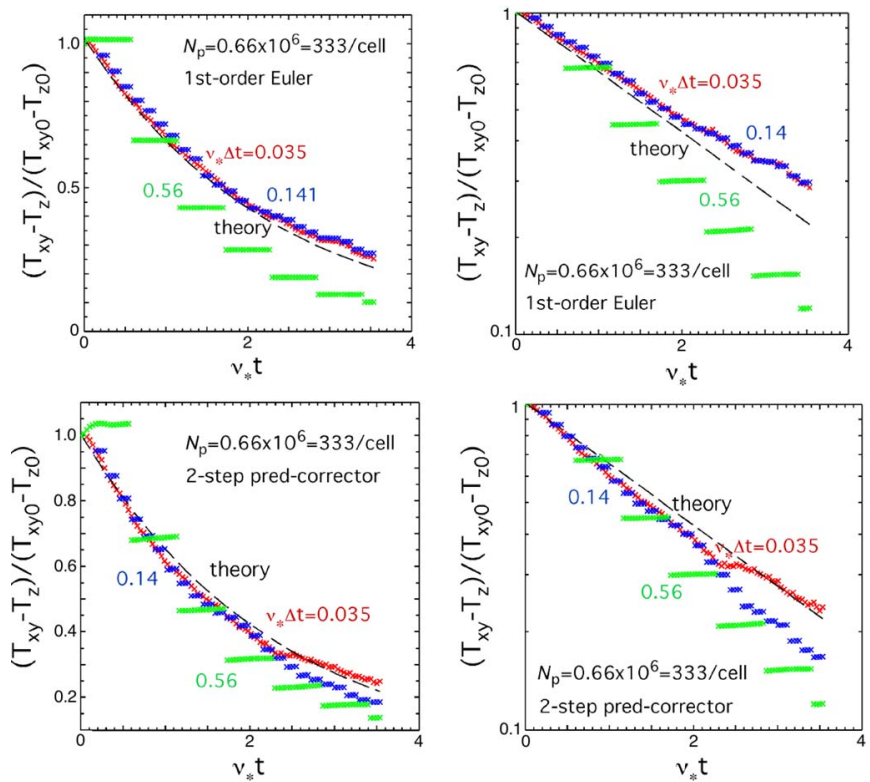

Fig. 5. BZOHAR simulations of like-species Coulomb collisions using the grid-based Langevin equations algorithm (1) with first-order Euler time integration and two-step predictor-corrector time integration to study the relaxation of a weak temperature anisotropy: simulated temperature anisotropy versus time for various values of the time step (linear and log-linear plots) (color online).

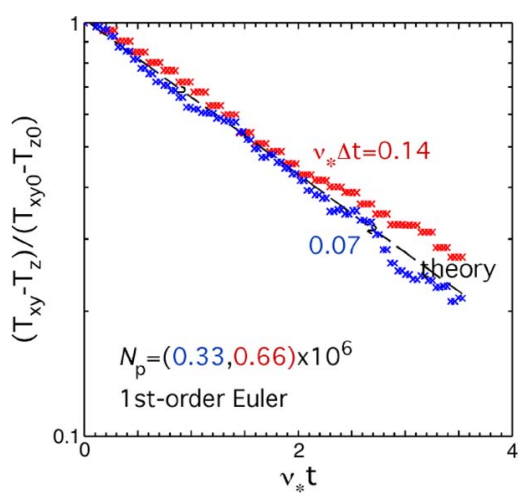

Fig. 6. BZOHAR simulations of like-species Coulomb collisions using the grid-based Langevin equations algorithm (1) with first-order Euler time integration to study the relaxation of a weak temperature anisotropy: simulated temperature anisotropy versus time for two values of the time step $\Delta t$ and particle number $N_{p}$ such that $N_{p} / \Delta t$ is held fixed (color online).

to the values of $\nu_{*} \Delta t$ considered. As the collisional time step is increased in the series shown in Fig. 5, the collision operator is applied with a discrete number of simulation time steps for the particle advection, electric field solve, and diagnostics in between collision operations, which accounts for the stair steps in the data shown in Figs. 4-10. Electric fields are calculated self-consistently at thermal levels, and particles are advected. The results from the two integration algorithms are similarly accurate, and the accuracy of the results degrades for $\nu_{*} \Delta t>$ 0.2 For $\nu_{*} \Delta t<0.1$, the relaxation agrees well with theory, and there is no obvious scaling of the results with $\nu_{*} \Delta t$. We note that in the convergence tests of the binary collision algorithm reported by Wang et al. [13], which address the same test problem, the relaxation of a weak temperature anisotropy, there is no clear scaling of errors with time step in some of the cases; and there is one example with scaling approximately
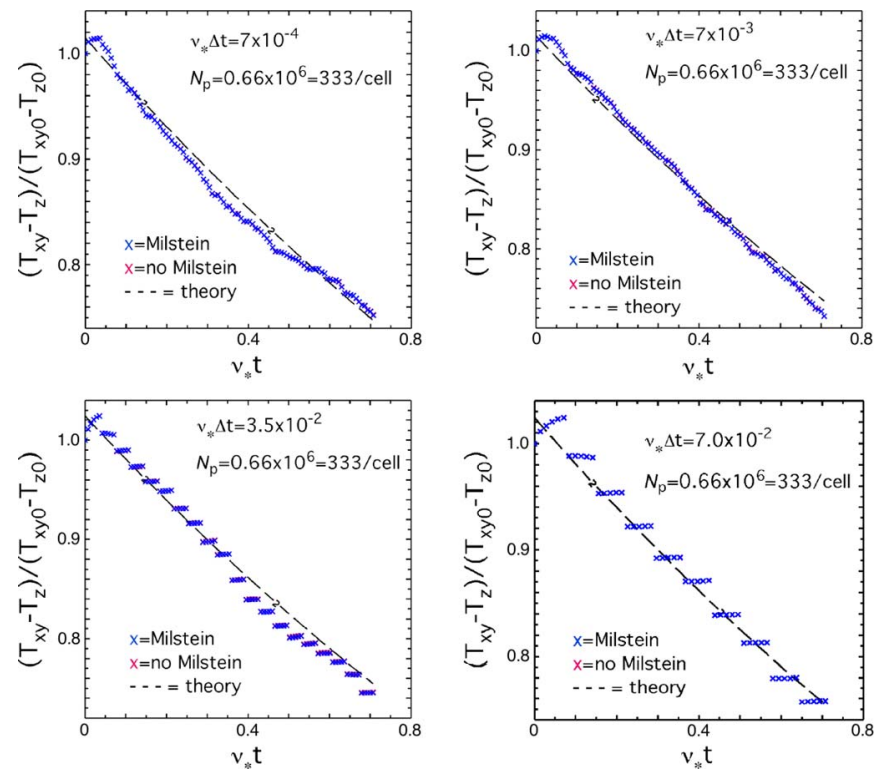

Fig. 7. BZOHAR simulations of like-species Coulomb collisions using the grid-based Langevin equations algorithm (1) with first-order Euler time integration and Milstein correction to study the relaxation of a weak temperature anisotropy: simulated temperature anisotropy versus time for four values of the time step $\Delta t$ and particle number $N_{p}$ (color online).

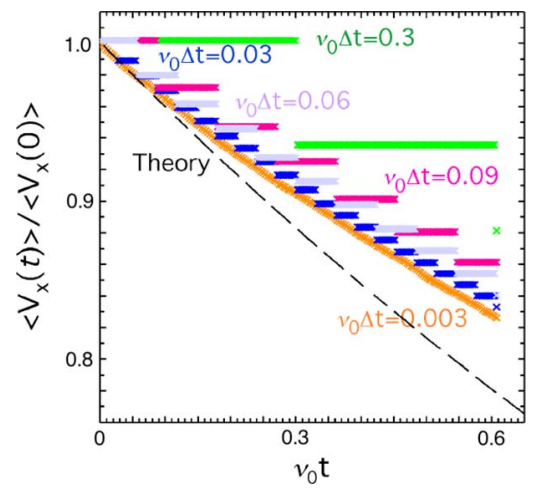

Fig. 8. Simulations of the relaxation of a relative electron-ion drift due to electron and ion collisions using the binary collision operator $\left\langle v_{x}(\mathrm{t})\right\rangle /\left\langle v_{x}(0)\right\rangle$ versus time for various values of the time step (color online).

proportional to $\Delta t^{1 / 2}$. There is no evidence of an error scaling that is proportional to $\Delta t$ or higher order in $\Delta t$ in the results for the Langevin-equations collision algorithms reported here and for the binary collision algorithm reported in [13].

We believe that the statistical errors dominate over the purely systematic errors associated with the discrete time integration of the collision operator for our parameters. If the finite number of collisions is a significant source of error in the collisional relaxation of the temperature anisotropy, then two simulations with the same number of total collisions proportional to the product of the total number of particles $N_{p}$ and the number of collisions each particle experiences, $\tau / \Delta t$ might yield similar error magnitudes. We illustrate this in Fig. 6, which shows the results of a simulation with $N_{p}=0.33 \times 10^{6}$ and $\nu_{*} \Delta t=0.07$ compared to a simulation with $N_{p}=0.66 \times 10^{6}$ and $\nu_{*} \Delta t=$ 0.14 The influence of thermal electric field fluctuations on the collisionality here is again small at $\nu_{i i}^{\text {eff }} \Delta t \leq 3.5 \times 10^{-4}$ because this is small compared to the values of $\nu_{*} \Delta t$ considered. 


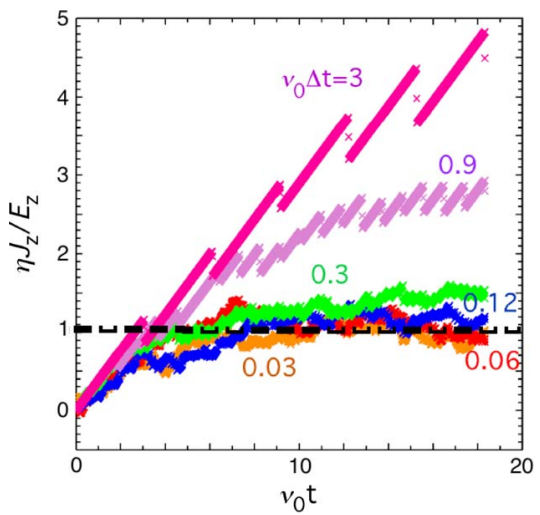

Fig. 9. Simulations of the relaxation of the plasma current $J_{z}$ to a resistive steady state in the presence of a weak, time-independent, and uniform electric field $E_{z}$ with binary electron and ion collisions $\eta J_{z} / E_{z}$ versus time for various values of the time step (color online).

The simulations use the first-order Euler grid-based Langevin equations collision algorithm (1) in one spatial dimension with self-consistent electric fields at thermal levels and including particle advection. Both simulations yield relatively good results as compared to the asymptotic theory, and the results exhibit similar error magnitudes.

Milstein [11], [12] has derived a subtle discrete time-step correction in the time integration of the drag-diffusion stochastic differential equations. Consider the first-order Euler representation of a stochastic differential equation

$$
\begin{aligned}
y_{n+1} & =y_{n}+a\left(y_{n}\right) \Delta t+b\left(y_{n}\right) \Delta W \\
\langle\Delta W\rangle & =0, \quad\left\langle\Delta W^{2}\right\rangle=\Delta t
\end{aligned}
$$

where $a$ represents the drag coefficient, $b$ is related to the square root of the diffusion coefficient, and $\Delta W$ is the normalized diffusive step size. The drag term on the right side is $O(\Delta t)$ and the diffusion term is $O\left(\Delta t^{1 / 2}\right)$. Given that $b$ is a function of the dependent variable $y$, which is evolving as a result of infinitesimal drag-diffusion incremental changes, Milstein observed that by iterating the Taylor-series expansion of the trajectory in the diffusive term, there is a correction to (12) at the same order as the drag term $b\left(y_{n}+O\left(\Delta t^{1 / 2}\right)\right) \Delta W=b\left(y_{n}\right) \Delta W+O(\Delta t)$. Generally, the stochastic kicks are intended to accumulate as a sum of infinitesimal kicks

$$
\begin{aligned}
\Delta y_{\text {stoch. }} & =\int_{t}^{t+\Delta t} d y=\int_{t}^{t+\Delta t} b(y) d \Delta W \\
& =b\left(y_{n}\right) \int_{t}^{t+\Delta t} d \Delta W+\left.\frac{d b}{d y}\right|_{y_{n}} \int_{t}^{t+\Delta t}\left(y-y_{n}\right) d \Delta W+\cdots \\
& \approx b\left(y_{n}\right) \Delta W+\left.b \frac{d b}{d y}\right|_{y_{n}} \int_{t}^{t+\Delta t} \Delta W d \Delta W+\cdots \\
& \approx b\left(y_{n}\right) \Delta W+\left.b \frac{d b}{d y}\right|_{y_{n}} \frac{1}{2}\left(\Delta W^{2}-\Delta t\right)+\cdots
\end{aligned}
$$

where we use $y_{n+1}-y_{n}=b \Delta W+$ higher order terms in $\Delta t$ and iterate the Taylor-series expansion. The expression

$$
\int_{t}^{t+\Delta t} \Delta W d \Delta W \approx \frac{1}{2}\left(\Delta W^{2}-\Delta t\right)
$$

derives from the use of Ito calculus [12] and follows from

$$
\begin{aligned}
\int_{0}^{t} W d W & =\sum_{j=1}^{n} W_{t_{j}}\left(W_{t_{j+1}}-W_{t_{j}}\right) \\
& =\frac{1}{2} W_{t}^{2}-\frac{1}{2} \sum_{j=1}^{n \rightarrow \infty}\left(W_{t_{j+1}}-W_{t_{j}}\right)^{2}=\frac{1}{2}\left(W_{t}^{2}-t\right) .
\end{aligned}
$$

Using (9), the drag-diffusion equation with Milstein correction becomes

$$
\begin{aligned}
y_{n+1}=y_{n}+a\left(y_{n}\right) \Delta t+b\left(y_{n}\right) \Delta W & \\
& +\left.\frac{1}{2} b \frac{d b}{d y}\right|_{y_{n}}\left(\Delta W^{2}-\Delta t\right) .
\end{aligned}
$$

The lowest-order Langevin equations (1) with Milstein correction at next order in $(\nu \Delta t)^{1 / 2}$ in the diffusion term for $v_{z}$ are then given by

$$
\begin{aligned}
& v_{z}^{t+\Delta t}=v_{z}^{t}+F_{d} \Delta t+g \Delta t^{1 / 2} N_{1}+\frac{1}{2} g \frac{d g}{d v} \Delta t\left(N_{4}^{2}-1\right) \\
& v_{\perp 1,2}^{t+\Delta t}=\Delta v_{\perp 1,2}
\end{aligned}
$$

where $\Delta \mathbf{v}_{\perp 1,2}$ are just the diffusion terms from (1). The Milstein corrections to $\Delta v_{\perp}$ at $O(\Delta t)$ enter at higher order in changing the particle energy

$$
v^{t+\Delta t}=\left[\left(v_{z}^{t}+\Delta v_{z}\right)^{2}+\Delta v_{\perp 1}^{2}+\Delta v_{\perp 2}^{2}\right]^{1 / 2}
$$

and are omitted. It is important to note that $\left\langle\Delta W^{2}-\right.$ $\Delta t\rangle=\left\langle N_{4}^{2}-1\right\rangle \Delta t=0$ so that the ensemble average of $\langle\Delta \mathbf{v} / \Delta t\rangle$ recovers the same Fokker-Planck drag coefficient and $\langle\Delta \mathbf{v} \Delta \mathbf{v} / \Delta t\rangle$ recovers the same diffusion tensor based on either (17) or (1), assuming that the random kicks are ideally distributed with zero mean and appropriately constrained variance.

In Fig. 7, we show the results of a series of simulations varying $\nu_{*} \Delta t$ over two orders of magnitude and comparing the results of the first-order Euler integration of (1) to the solution of the difference equations, including the Milstein correction in (17) for the relaxation of a small temperature anisotropy, as in Figs. 1-3. The influence of thermal electric field fluctuations on the collisionality in this series of simulations is mostly negligible, except at the smallest value of $\nu_{*} \Delta t$ used: $\nu_{i i}^{\text {eff }} \Delta t \sim$ $2 \times 10^{-4}<\nu_{*} \Delta t$. The Milstein corrections are nonzero, but are so small that the results almost overlay one another in the plots. The smallness of the Milstein corrections for Coulomb collisions in this example derives partly from the smallness of $d g / d v$ over most of velocity space, except for test-particle 

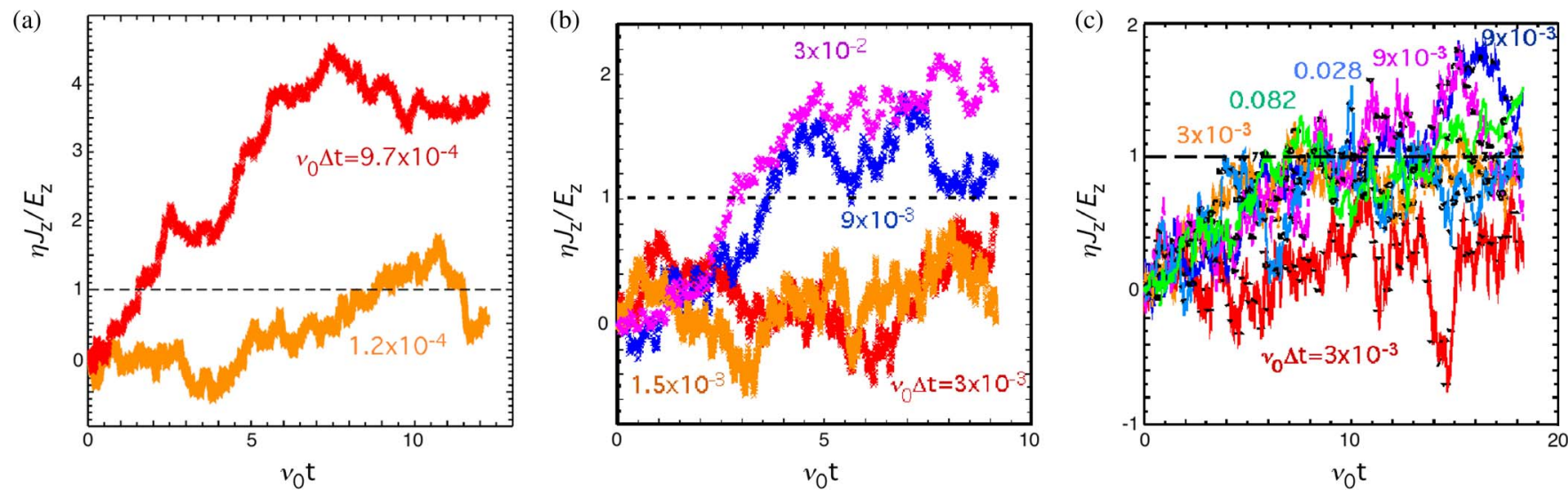

Fig. 10. Simulations of the relaxation of the plasma current $J_{z}$ to a resistive quasi-steady state in the presence of a weak, time-independent, and uniform electric field $E_{z}$ with grid-based Langevin equations for electron and ion collisions: $\eta J_{z} / E_{z}$ versus time for various values of the time step and algorithms based on (a) (1), (b) (3), and (c) (3), with modifications for either energy and momentum conservation or electron-ion pitch-angle collisions or increased number of particles. (See text for definitions of the color traces; color online.)

velocities that are comparable to the thermal speed of the field particles. This significantly limits the influence of the Milstein correction on the calculation of kinetic energy moments of the distribution function which sample all of the velocity space. Similarly, small Milstein corrections have been reported by Lemons et al. [6] in their simulations of Coulomb collisions.

The derivation of the Milstein correction illustrates the $\Delta t$ accuracy of the collision model based on the Langevin equations. The Langevin equation, e.g., (8) or (12), is a stochastic differential equation whose next order in powers of $\Delta t^{1 / 2}$ correction is proportional to $\Delta t\left(N_{4}^{2}-1\right)$ shown in (17), where $N_{4}$ is a random number sampled from an ideal Gaussian distribution with zero mean and a variance equal to unity. Of course, any finite sample of random numbers has a sample mean and sample variance that differ in general from the mean and variance of the parent distribution. Without the Milstein correction, the stochastic differential equation is accurate through $O\left(\Delta t^{1 / 2}\right)$. Consider the diffusion term in (8) containing $\Delta t^{1 / 2} N_{1} \cdot N_{1}$ is also a random number sampled from a finite set of $N_{r}$ random numbers that belong to a parent distribution that is Gaussian. Because the variance of $\left\{N_{1}\right\}$ differs from unity in general, the time-averaged numerical diffusion rate acquires a random error and is not exactly consistent with the desired diffusion coefficient. Statistical theory asserts that the variance of the collisional velocity changes proportional to $\Delta t^{1 / 2} N_{1}$ sampled over a time interval (and hence the timeaveraged numerical diffusion coefficient) will have a relative standard error whose size scales as $1 / N_{r}^{1 / 2}$. With one collision per particle per time step, the number of collision events in a physical time interval $\tau$ will be proportional to $\tau / \Delta t$; hence, the relative random error in the numerical diffusion will scale as $\Delta t^{1 / 2}$ over a physical time interval. Thus, there is no clean separation between the purely systematic $\Delta t$ errors in the integration of the Langevin equations and the random errors associated with the collisions. These random errors affect the time histories of the collisional relaxation processes. In contrast, the lowest-order Euler integration of a nonstochastic differential equation is accurate through $O(\Delta t)$, with errors at $O\left(\Delta t^{2}\right)$. The arguments presented here provide insight into the findings in the simulations that the random errors are dominant.

\section{Particle Simulation of ELECTRON-ION COLLISIONS}

Electron-ion collision processes must be accurately resolved in kinetic simulations to reproduce faithfully the classical plasma resistivity. Classical resistivity is an important ingredient in the quasi-neutral response of a high-density collisional plasma to injected beams and driving electric fields. What are the requirements on time step for resolving electron-ion collisions in a particle simulation? We have studied (1) the relaxation of a relative drift between electrons and ions using binary collisions and (2) the approach to a steady-state resistive response to a weak driving electric field using both the binary collision algorithm and the grid-based Langevin equations collision algorithms.

For a drifting Maxwellian electron velocity distribution, the initial slowing-down rate for electrons on ions with $T_{i} \ll$ $\left(m_{i} / m_{e}\right) T_{e}$ is given by [1]

$$
\begin{aligned}
& \nu_{s}=\mu\left(\varepsilon / T_{e 0}\right)\left(T_{e 0} / \varepsilon\right)^{3 / 2} \nu_{0} \\
& \nu_{0}=4 \pi n e^{4} \ln \Lambda / 2 \sqrt{2} m_{e}^{1 / 2} T_{e}^{3 / 2}, \quad \text { for } Z=1 \text { and } T_{e}=T_{i} \\
& \mu=\left(2 / \pi^{1 / 2}\right) \int_{0}^{x} e^{-\xi} \xi^{1 / 2} d \xi=\operatorname{erf}(x)-\left(2 / \pi^{1 / 2}\right) x e^{-x} \\
& x=\left(\varepsilon / T_{e 0}\right)^{1 / 2}
\end{aligned}
$$

where $\varepsilon=m_{e} v_{d}^{2} / 2$ is the kinetic energy associated with the initial electron drift and $\mu=0.428$ for $x=1$. The slowing down of electrons is studied in a series of initial-value simulations using the binary collision algorithm varying $\nu_{0} \Delta t$ with initial conditions $\varepsilon_{0}=m_{e} v_{d 0}^{2} / 2=T_{e 0}=T_{\mathrm{i} 0}$. Good results for the initial relaxation rate are obtained for $\nu_{0} \Delta t<0.1$ (Fig. 8). In these simulations, electron-electron, electron-ion, and ion-ion collisions were simulated in a 1-D periodic domain with the electric field suppressed and with $m_{i} / m_{e}=1836$ and $Z_{i}=1$. In [2], the analysis of the slowing down of a drifting electron Maxwellian in the presence of only electron-ion collisions is extended to finite time durations by invoking energy conservation 
for the electrons (valid for $m_{i} / m_{e} \gg 1$ ) so that $\varepsilon+3 T_{e} / 2=$ const. for all time, replacing the relaxation rate with the timedependent expression $\nu_{s}=\mu\left(\varepsilon / T_{e 0}\right)\left(T_{e 0} / \varepsilon\right)^{3 / 2} \nu_{0}$ and integrating the relaxation equation. This allows the instantaneous exponential relaxation rate to evolve for finite time and gives the theoretical curve shown in Fig. 8 a decreasing slope. There is a second mechanism that can contribute to a reduction in the exponential relaxation rate for finite time. In the smallmass-ratio limit, the slowing down of a drifting Maxwellian test-particle population can be evaluated using the analytical solution for the evolution of a distribution function under Coulomb-Lorentz collisions given in [9]. The analysis shows that there is a decrease in the exponential relaxation rate as a function of time, which derives from the more energetic electrons taking a longer time to collisionally relax than the less energetic electrons. This then produces a deviation of the electron velocity distribution from a Maxwellian. Such a calculation was given for temperature-anisotropy relaxation in [15]. The decrease in the slowing-down rate after $\nu_{0} t \approx 0.1$ in the smallest time-step case is qualitatively consistent with the analytically determined behavior for the test-electron slowingdown problem.

The approach to a resistive quasi-steady state was studied in a series of simulations with the binary and gridbased Langevin equations collisions algorithms to compare their performance. For a small constant applied small electric field, $E_{z}=0.6 \mathrm{~V} / \mathrm{cm},\left(e / m_{e}\right) E_{z} \Delta t^{2} / \Delta x=7 \times 10^{-7}$ and $\left(e / m_{e}\right) E_{z} \Delta t /\left\langle v_{z 0}^{2}\right\rangle^{1 / 2}=4 \times 10^{-5}$, with $m_{i} / m_{e}=1836$, binary collisions, $T_{e}=T_{i}$, and $5 \times 10^{4}$ particles of each species, we observe in Fig. 9 that the plasma acquires a quasi-steady current in a time $\sim 5 \nu_{0}^{-1}$, which satisfies the resistive relation [20]

$$
E_{z}=\eta_{\|} J_{z}, \quad \eta_{\|}=0.51 \frac{m_{e}}{e} \frac{1}{n_{e} e \tau_{e}}, \quad 1 / \tau_{e}=(4 / 3 \sqrt{\pi}) \nu_{e i}
$$

where $\nu_{e i}=\sqrt{2} \pi Z_{i}^{2} e^{4} n_{i} \ln \Lambda / \sqrt{m_{e}} T_{e}^{3 / 2}$. The relaxation is studied in the numerical examples varying $\nu_{0} \Delta t$, and relatively good results are obtained for $\nu_{0} \Delta t \leq 0.1$ There are no self-consistent thermal electric field fluctuations in the simulations shown in Figs. 9 and 10. The constant applied electric field in this example must be small enough so that the acceleration and resistive heating of the electrons is weak over the duration of the simulation. In general, as the plasma electrons heat, the resistivity will decrease as $T_{e}$ increases (not allowed in our simulation diagnostic) and the plasma current is expected to increase. Consequently, high-energy electrons can run away if the electric field is too large. We observe electron velocity distributions that evolve in the presence of the applied electric field to form high-energy tails because they are more weakly collisional and more easily accelerated. This is how "slide-away" velocity distribution functions are formed in the presence of accelerating electric fields. The collisions tend to isotropize the electron velocity distribution.

Simulations studying the approach to a resistive quasi-steady state using the grid-based Langevin equations collision algorithms are shown in Fig. 10. In the simulations in Fig. 10(a) and (b), $E_{z}=0.3 \mathrm{~V} / \mathrm{cm},\left(e / m_{e}\right) E_{z} \Delta t^{2} / \Delta x=3.5 \times 10^{-7}$, and $\left(e / m_{e}\right) E_{z} \Delta t /\left\langle v_{z 0}^{2}\right\rangle^{1 / 2}=2 \times 10^{-5}$, and we used $4 \times 10^{4}$ particles of each species with $T_{e}=T_{i}, m_{i} / m_{e}=1836$ and singly charged ions. Using (1), the expected relaxation is reproduced in Fig. 10(a) for $\nu_{0} \Delta t=1.2 \times 10^{-4}$ over $10^{5} \Delta t$ with a $3 \%$ growth of the total kinetic energy, and poor results are obtained with $\nu_{0} \Delta t=9.7 \times 10^{-4}$ with a significant loss of energy conservation: there is a three-fold growth of the total kinetic energy after $10^{4} \Delta t$. A much smaller time step, $O\left(10^{-3}\right)$ smaller, must be used here than with the binary collision operator in keeping with the arguments in Section II. Using (3), the relaxation agrees adequately with theory for $\nu_{0} \Delta t \leq 9 \times 10^{-3}$ and departs from theoretical expectation for $\nu_{0} \Delta t \geq 3 \times 10^{-2}$ with more than a two-fold growth of the total kinetic energy in $3 \times 10^{3} \Delta t$. Thus, the Lemons et al. algorithm (3) allows the use of a $\nu_{0} \Delta t$ value that is $O\left(m_{i} / m_{e}\right)^{1 / 2}$ larger than for the algorithm based on (1) in this example. However, the value of $\nu_{0} \Delta t$ can be chosen an order of magnitude larger for the binary collision algorithm compared to that using the Lemons $e t$ al. algorithm in Fig. 10(b).

In Fig. 10(c), additional results are shown for the numerical simulation of the approach to a resistive quasi-steady state using the Lemons et al. algorithm (3) with various modifications to the algorithm or changes in the parameters, e.g., changing the time step or the number of particles. One can modify the collision algorithm to enforce momentum and kinetic energy conservation. After each like-particle collision, the momenta are uniformly shifted in each cell (or globally if a uniform plasma problem is being studied, as is the case here) and the momenta are uniformly scaled relative to the average momentum in the cell to conserve momentum and energy [4], [6]. For unlikeparticle collisions, e.g., electron-ion or ion-electron collisions, the changes in the local (or global) total momentum and kinetic energy of the test particles can be compensated for by equal and opposite changes in the field particles momenta and kinetic energies by means of locally uniform momenta shifts and scaling of the momenta relative to the average momentum of the field particles so that the sum of the total momentum and kinetic energy of the test and field particles is conserved. For small time steps and good statistics, the momenta shifts are small and the scaling is near unity. Alternatively, we could shift the momenta of both the test and field particles to conserve total momentum, and then scale both the test and field particle momenta relative to the mean momenta of each species to conserve total energy. The results in Fig. 10(c) show no significant improvement in the relaxation to a resistive steady state compared to the results in Fig. 10(b) due to restoring momentum and energy conservation after the Monte Carlo collisions. However, doubling the number of particles for an adequately small time step results in a significant improvement in the comparison of the relaxation to the theoretical steady state in Fig. 10(c).

The traces in Fig. 10(c) are labeled as follows: red = \{Eq. (3) with $\nu_{0} \Delta t=3 \times 10^{-3}, N_{p}=40000$ for each species, with adjusted energy/momentum conservation $\}$; blue $=$ \{Eq. (3) with $\nu_{0} \Delta t=9 \times 10^{-3}, N_{p}=40000$ for each species, with adjustments for energy/momentum conservation $\}$; coral $=$ \{Eq. (3) with $\nu_{0} \Delta t=3 \times 10^{-3}, N_{p}=80000$ for each species, and no adjustment for energy/momentum conservation $\}$; magenta $=\{$ Eq. (3) with electron-ion collisions reduced to pitchangle collisions, $\nu_{0} \Delta t=9 \times 10^{-3}, N_{p}=40000$ for each 
species, and no adjustment for energy/momentum conservation $\}$; light blue $=\{$ Eq. (3)with electron-ion collisions reduced to just pitch-angle collisions, $\nu_{0} \Delta t=0.028, N_{p}=40000$ for each species, and no adjustment for energy/momentum conservation $\}$; and green $=\{$ Eq. (3) with electron-ion collisions reduced to just pitch-angle collisions, $\nu_{0} \Delta t=0.082, N_{p}=80000$ for each species, and no adjustment for energy/momentum conservation $\}$.

The reduction of the Lemons et al. algorithm (3) to just pitch-angle collisions is shown to be particularly effective in the results shown in Fig. 10(c). To achieve this reduction in (3), one suppresses the drag and diffusion of the particle speed, i.e., one forces $\Delta v=0$ for collisions of electrons on ions, which is a very good approximation for $m_{e} / m_{i} \ll 1$. Only pitch-angle collisions of the electrons survive in this reduction. For larger values of $\nu_{0} \Delta t$, if an electron undergoes a largeangle scattering event, it still conserves energy. Like the binary collision algorithm, the accuracy of the collision algorithm fails gracefully. Data for the relaxation to a resistive steady state is shown in Fig. 10(c) extending to much larger values of $\nu_{0} \Delta t$ than in Fig. 10(b), and good results are recovered compared to the theory provided that there is adequate statistical resolution of the collisions. Energy conservation with no modification of the algorithm for energy/momentum conservation after the inter-species collisions is within a few percent over 6000 time steps. The results for the relaxation to a resistive steady state using the pitch-angle limit of (3) for electron-ion collisions are similar to those obtained using the binary collision operator shown in Fig. 9 with respect to time step, but the binary collision algorithm yields smoother time traces.

\section{SUMmARY}

We have studied some of the accuracy issues for gridbased Langevin equations and binary collision algorithms. In an example of practical interest using numerical parameters that are typical of plasma physics simulations using first-order Euler and higher-order time-integration algorithms for the gridbased Langevin equations collision model based on (1), we find that statistical noise errors associated with finite numbers of particles and collision events are dominant sources of error in the computations. We have argued that the statistical noise errors in the collision events can be, in general, conflated with time-step issues. The dependence of the results on the time step in our simulation examples is weak for the test case of the relaxation of a weak temperature anisotropy. For the parameters employed in our simulations, there is no improvement in the results achieved with higher-order-accurate time-integration schemes, and acceptable accuracy can be obtained with a firstorder Euler time integration for an appropriate choice of time step. The numbers of particles per cell and total numbers of particles used in the simulations are representative of wellresolved particle simulations commonly employed to study plasma phenomena. We obtain similarly good results on this same relaxation problem using a first-order Euler integration of the Lemons et al. algorithm (3) for values of $\nu_{*} \Delta t<0.1$. Results for the same collisional relaxation problem using the binary collision algorithm have been reported by Wang et al. [13], who also observed a weak dependence of the accuracy of the results on the time step. We also find that when a Milstein correction [11], [12] to the Langevin equations model is included, there is very little change in the observed results for the collisional relaxation of a weak temperature anisotropy studied here. The Milstein correction analysis also illustrates the dependence of the errors in the integration scheme on $\Delta t$ and the sampling of the random numbers. The analysis of the Milstein correction and the arguments on the scaling of the random errors presented in Section III support the simulation findings that the random errors dominate any systematic time step errors.

We have not investigated the convergence of the simulations of collision processes studied here with respect to numbers of particles or ensembles of realizations of the random numbers in the collision operators. Achieving improved statistical resolution to a high degree can be challenging. To reduce the stochastic statistical errors by $O(1 / 10)$ would require $O\left(10^{2}\right) \times$ more particles and collision events per particle per time step given the $1 / N^{1 / 2}$ scaling of the statistical errors, which could be impractical on a routine basis. Moreover, if the number of collisions per particle per time step remains fixed at unity, a decrease in the collisonal time step by $1 / 10^{2}$ would be required in order to increase the number of collision events by $10^{2}$ over a defined time interval. Here, we are again confronted with the conflation of statistical errors with the time step used in the integration. In the multilevel Monte Carlo methodology suggested by M. B. Giles [21], the linkage of the number of random collision events per particle per time step is relaxed by averaging over additional collisional integration paths with different values of the time step. Giles provides estimates of the statistical error due to the sampling of random numbers in the collision events and the integration error due to $\Delta t$, and he shows how to balance the two sources of error while minimizing the computational burden. However, Giles' method has not as yet been applied to simulating collisions in plasmas.

We have also studied the collisional relaxation of an initial electron-ion relative drift and the relaxation to a resistive steady-state in which a quasi-steady current is driven by a weak applied electric field, as functions of the time step used to resolve the collision processes. For a sufficiently small time step, the particle simulations recover the initial relaxation rate for an electron-ion relative drift [1] and the classical resistivity predicted by Braginskii's theory [20]. Because the grid-based Langevin equations algorithm in (1) has an unfavorable massratio scaling at low test-particle velocities, modeling electronion collisions with this algorithm requires a much smaller time step than that required using the algorithm based on (3) or the Takizuka and Abe binary collision algorithm. A significantly larger collisional time step can be used with the binary collision algorithm than with either of the unmodified Langevin equations collision algorithms for the example of the relaxation to a resistive steady state involving electron-ion collisions investigated here. However, if the Lemons et al. algorithm (3) is reduced to just pitch-angle scattering for electron-ion collisions, which is a good approximation for $m_{e} / m_{i} \ll 1$, then the results for the relaxation to a resistive steady state are nearly as good as 
the results from the binary collision operator. Both algorithms are shown to extend to larger values of the time step.

The binary collision model and the grid-based Langevin equations collision models are widely used, and good results can be obtained with both approaches. The binary collision model requires some additional computations to do the sorting and pairing of particles in a spatial cell as compared to the Langevin equations model. Moreover, the latter model is more easily parallelized. However, the binary collision model described in [1] and [2] naturally conserves momentum and energy. The Langevin equations model is more restrictive with respect to the assumption that the velocity distribution function of the field particles is close to a Maxwellian (although this assumption can be relaxed if one is willing to calculate new drag and diffusion coefficients from the Rosenbluth potentials [4], [14]). In our experience, we have used both approaches and advocate letting the nature of the problem at hand drive the choice of which approach to use.

Although the results presented here are not intended to be a detailed convergence analysis of collision algorithms in particle codes, significant insights are presented into the characteristics of the collision algorithms and their performance. The simulation results presented here should be of value in providing guidance in choosing between the collision algorithms and selecting time steps for resolving collisional relaxation processes in particle simulations.

\section{ACKNOWLEDGMENT}

The authors would like to thank A. Kemp, D. Lemons, D. Strozzi, D. Grote, and C. M. Wang for useful discussions and suggestions.

\section{REFERENCES}

[1] T. Takizuka and H. Abe, "A binary collision model for plasma simulation with a particle code," J. Comput. Phys., vol. 25, no. 3, pp. 205-219, Nov. 1977.

[2] K. Nanbu, "Theory of cumulative small-angle collisions in plasmas," Phys. Rev. E, Stat. Phys. Plasmas Fluids Relat. Interdiscip. Top., vol. 55, no. 4, pp. 4642-4652, Apr. 1997.

[3] M. E. Jones, D. S. Lemons, R. J. Mason, V. A. Thomas, and D. Winske, "A grid-based Coulomb collision model for PIC codes," J. Comput. Phys., vol. 123, no. 1, pp. 169-181, Jan. 1996.

[4] W. M. Manheimer, M. Lampe, and G. Joyce, "Langevin representation of Coulomb collisions in PIC simulations," J. Comput. Phys., vol. 138, no. 2, pp. 563-584, Dec. 1997.

[5] B. I. Cohen, L. Divol, A. B. Langdon, and E. A. Williams, "Effects of ion-ion collisions and inhomogeneity in two-dimensional kinetic ion simulations of stimulated Brillouin backscattering," Phys. Plasmas, vol. 13, no. 2, p. 022705 , Feb. 2006.

[6] D. S. Lemons, D. Winske, W. Daughton, and B. Albright, "Small-angle Coulomb collision model for particle-in-cell simulations," J. Comput. Phys., vol. 228, no. 5, pp. 1391-1403, Mar. 2009.

[7] M. Sherlock, "A Monte-Carlo method for Coulomb collisions in hybrid plasma models," J. Comput. Phys., vol. 227, no. 4, pp. 2286-2292, Feb. 2008.

[8] L. Spitzer, The Physics of Fully Ionized Gases. New York: Wiley Interscience, 1956.

[9] B. A. Trubnikov, "Particle interactions in a fully ionized plasma," in Reviews of Plasma Physics. New York: Consultants Bureau, 1965, p. 105.

[10] T. D. Rognlien and T. A. Cutler, "Transition from Pastukhov to collisional confinement in a magnetic and electrostatic well," Nucl. Fusion, vol. 20, no. 8, pp. 1003-1011, Aug. 1980.

[11] G. N. Milstein, "Approximate integration of stochastic differential equations," Theory Probab. Appl., vol. 19, no. 3, pp. 557-562, Jun. 1975.
[12] P. E. Kloeden and E. Platen, Numerical Solution of Stochastic Differential Equations. Berlin, Germany: Springer-Verlag, 1999, sec. 10.3.

[13] C. M. Wang, T. Lin, R. Caflisch, B. I. Cohen, and A. M. Dimits, "Particle simulation of Coulomb collisions: Comparing the methods of Takizuka and Abe, and Nanbu," J. Comput. Phys., vol. 227, no. 9, pp. 4308-4329, Apr. 2008.

[14] M. N. Rosenbluth, W. M. MacDonald, and D. L. Judd, "Fokker-Planck equation for an inverse-square force," Phys. Rev., vol. 107, no. 1, pp. 1-6, Jul. 1957.

[15] A. M. Dimits, C. Wang, R. Caflisch, B. I. Cohen, and Y. Huang, "Calculation of Trubnikov and Nanbu collision kernels: Implications for numerical modeling of Coulomb collisions," J. Comput. Phys., vol. 228, pp. 48814892, Apr. 2009.

[16] A. Friedman, "Partially-corrected Euler method for solution of ODE's," Lawrence Berkeley Nat. Lab., Berkeley, CA, Tech. Rep. UCRL-TR235096, Sep. 28, 2007.

[17] C. K. Birdsall and A. B. Langdon, Plasma Physics via Computer Simulation. New York: McGraw-Hill, 1985.

[18] H. X. Vu, D. F. DuBois, and B. Bezzerides, "Inflation threshold; A nonlinear trapping-induced threshold for the rapid onset of stimulated Raman scattering from a single laser speckle," Phys. Plasmas, vol. 14, no. 1, p. 012702 , Jan. 2007.

[19] B. I. Cohen, H. X. Vu, and E. A. Williams, "Kinetic-ion simulations addressing whether ion trapping inflates stimulated Brillouin backscattering reflectivities," Phys. Plasmas, vol. 14, no. 10, p. 102 707, Oct. 2007.

[20] S. I. Braginskii, "Transport processes in a plasma," in Reviews of Plasma Physics. New York: Consultants Bureau, 1965, p. 205.

[21] M. B. Giles, "Multilevel Monte Carlo path simulation," Oper. Res. vol. 56, no. 3, pp. 607-617, May/Jun. 2008.

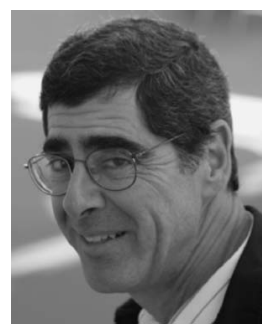

Bruce I. Cohen received the Ph.D. degree in plasma physics from the University of California, Berkeley, in 1975.

He worked at Princeton Plasma Physics Laboratory, Princeton, NJ, as a Postdoctoral Researcher from 1975 to 1976 . He is currently a Research Physicist and the Associate Program Leader of the Fusion Energy Theory and Computations Program, Lawrence Livermore National Laboratory, Livermore, CA. His research interests are in magnetic and inertial confinement fusion and computational plasma physics.

Dr. Cohen is a Fellow of the American Physical Society elected through the Division of Plasma Physics.

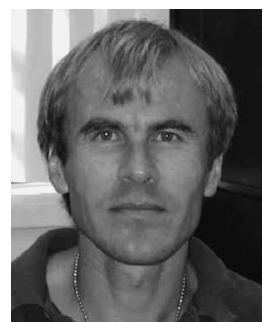

Andris M. Dimits received the Ph.D. degree in astrophysical sciences from Princeton University, Princeton, NJ, in 1988

He worked at the University of Maryland, College Park, as a Postdoctoral Researcher from 1988 to 1990 . He is currently a Research Physicist at the Fusion Energy Theory and Computations Program, Lawrence Livermore National Laboratory, Livermore, CA. His research interests are in magnetic fusion plasmas; nonlinear plasma dynamics; kinetic and fluid simulation techniques for plasmas, including derivation; implementation and application of gyrokinetic models; direct simulation and turbulence closures for fluids; and high-energy density physics, including instabilities in radiative hydrodynamics. He is one of the originators of the $\delta f$ method. 


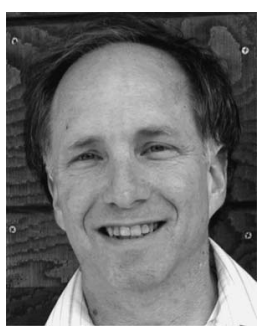

Alex Friedman received the Ph.D. degree in applied physics from Cornell University, Ithaca, NY.

He then carried out Postdoctoral Research at the University of California, Berkeley. In 1980, he joined the staff of Lawrence Livermore National Laboratory (LLNL), Livermore, CA, where he is an Associate Program Leader of the Fusion Energy Program. His research interests include heavy-ion beam-driven inertial fusion energy, computational plasma physics and particle-beam physics, computational dynamics, and numerical analysis.

Dr. Friedman is a Fellow of the American Physical Society and a recipient of the LLNL Physics Department's Distinguished Achievement Award. He currently serves as the Simulations and Theory Group Leader of the Virtual National Laboratory for Heavy Ion Fusion Science, a collaboration of Lawrence Berkeley National Laboratory, Lawrence Livermore National Laboratory, and the Princeton Plasma Physics Laboratory.

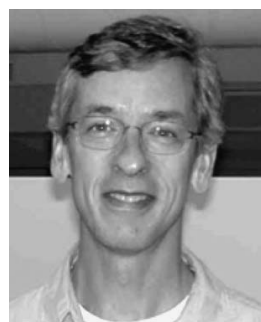

Russel E. Caflisch received the Ph.D. degree in mathematics from the Courant Institute of Mathematics, New York University, New York, in 1978.

He has held faculty positions at NYU and Stanford University. Since 1989, he has been a Professor in the Mathematics Department of the University of California at Los Angeles, Los Angeles, where he is also Director of the Institute for Pure and Applied Mathematics. His research interests are in computational and applied mathematics for fluids, plasmas, materials, and finance.

Dr. Caflisch is a Fellow of the Society for Industrial and Applied Mathematics. 